\title{
Surveillance of Mosquitoes for Zika Virus in Westchester County, New York
}

\author{
Randolph Earl Schilke \\ New York Medical College, Valhalla, New York, United States; 40 Sunshine Cottage Rd., Valhalla, NY 10595, \\ USA; Randolph_Schilke@nymc.edu; Tel.: +1-734-904-6610
}

\begin{abstract}
In 2015 in Brazil, Zika virus showed features of geographic expansion and potentially increased virulence. In 2016, New York State issued emergency regulations after the World Health Organization declared Zika virus a Public Health Emergency of International Concern. In this study, mosquito surveillance was conducted in Westchester County, New York, to identify Zika virus and other arboviruses. Twenty trap sites were used for surveillance of Aedes albopictus mosquitoes, the Zika virus vector. The Westchester County Department of Health performed testing for Zika, West Nile, Eastern equine encephalitis, and other flaviviruses on 369 batches comprising 8,891 mosquitoes. Aedes albopictus mosquitoes were identified in Nassau, Rockland, Suffolk, Westchester, and New York City. Despite the increased capacity for specimen analysis, Zika virus was not detected. This study provides the first evidence of appropriate Zika virus surveillance. However, the results do not allow determination of the potential mechanism of local vector-to-human transmission of Zika virus among Aedes albopictus mosquitoes. This study adds to the evidence regarding the distribution, emergence, and trapping capabilities of potential Zika virus vectors.
\end{abstract}

Keywords: Zika virus; Flavivirus; RNA Viruses; Viruses; surveillance; occurrence; epidemiology; West Nile virus; Aedes; Culicidae

\section{INTRODUCTION}

Zika virus (ZIKV) was first identified in 1947 in the blood of rhesus monkeys in the Zika forest in Uganda. ZIKV is a flavivirus related to Dengue, West Nile virus (WNV), Yellow fever, and Japanese encephalitis [1]. Primary vector-to-human transmission is speculated to occur from mosquitoes of the Aedes (A.) genus, specifically, A. aegypti [2-3] and A. albopictus [4]. During the decades preceding the discovery of ZIKV, documented serologic evidence of human infections occurred in several countries in Africa, Asia, and Micronesia, the most common clinical characteristics of which included macular rash, fever, arthritis, conjunctivitis, myalgia, headache, edema, and vomiting [5]. More recently, rapid geographic expansion and potential increases in the virulence of Zika were noted during the 2015-2016 Brazilian ZIKV outbreak, with some evidence suggesting that the virus may result in impaired fetal brain development and Guillain-Barré syndrome [6]. Although documented transmission had occurred throughout Africa, Asia, and Micronesia during most of the 20th century, ZIKV-related infections were not associated with any life-altering human pathology until 2015 [7].

On February 1, 2016, the World Health Organization declared ZIKV a Public Health Emergency of International Concern due to a suspected increase in the rate of microcephaly among newborns with Zika. The State of New York issued emergency regulations, effective March 17, 2016, that amended Title 10 of the New York Codes, Rules, and Regulations (10 NYCRR) Subpart 40-2 by adding a new section-10 NYCRR 40-2.24-in response to the emergence and worldwide public health threat of ZIKV. This new section requires local health departments to adopt and implement a Zika Action Plan (ZAP), using guidance issued by New York State Department of Health (NYSDOH). As per Westchester County Department of Health (WCDH) and NYSDOH, ZAP is an integral component of disease prevention that incorporates timely human illness monitoring, surveillance via mosquito collection, and rapid dissemination of results. 
In the present study, the WCDH aimed to determine whether ZIKV was present in mosquitoes collected during the summer of 2016, in Westchester County, New York. This report provides the first evidence of ZIKV surveillance activity in southern New York via appropriate field assessment involving trapping, sampling, and testing of local mosquito populations. It also reports the first known distribution of $A$. albopictus by BG-Sentinel 2 traps in southern New York, providing clinicians and public health officials with critical information regarding the potential geographic expansion of possible ZIKV vectors.

\section{MATERIALS AND METHODS}

\subsection{Trapping Mechanisms}

The WCDH used three types of traps during vector surveillance: BG-Sentinel 2 trap (BG trap), Centers for Disease Control and Prevention Light Trap (CDC light trap), and a Frommer Updraft Gravid Trap (Gravid trap). This study had access to six BG traps that used an attractant produced by BioGents [8]. A 12V/14-Ahr BioQuip DC battery pack (Model 2861) was used to power each BG trap. Twenty CDC light traps were baited with $\mathrm{CO}_{2}$ via a $1.9 \mathrm{~L}$ cooler with a 0.125 -bit hole drilled in the bottom that had been filled with dry ice. A 6V Powersonic battery (Model PS-6100F1) was used to power each CDC light trap. Gravid traps were baited using fermented hay and water and also powered using a 6V Powersonic battery (Model PS-6100F1).

\subsection{Trap Locations}

Active adult mosquitoes were collected from 20 sites throughout Westchester County, including urban and rural habitats and differing types of forest (Figure 1). As shown in Figures 1 and 2, six of ten existing trap locations had both a CDC light trap and a Gravid trap; four other existing trap sites, all stationed in southern Westchester County - where the WCDH has historically collected the largest number of A. albopictus specimens - had three traps: a CDC light trap, a Gravid Trap, and a BG trap. Ten new sites, each having a CDC light trap and a Gravid trap, were added to increase the geographic distribution. Two of these new sites were supplemented with BG traps in August 2016.

\subsection{Mosquito Trapping}

Mosquito trapping occurred from June 2016 through to the end of September 2016. Traps were set before midday (12:00 PM) and collected the following day at daybreak, Monday through Thursday. Traps were collected from the field sites, placed in coolers with dry ice, and transported back to the WCDH Mosquito Laboratory, where they were then placed in a freezer. Mosquitoes from each trap and location were then sub-sorted according to sex, and the male mosquitoes were discarded. Females were classified according to species and grouped into batches containing 10-60 individual specimens of the same species, site, location, and week of collection. Attempts were made to distinguish $C$ ulex $(C x$.) pipiens and $C x$. restuans mosquitoes as separate species. Unfortunately, these attempts were abandoned because the majority of samples were extensively damaged; thus these mosquito samples were not tested separately but were combined into one species as $C x$. pipiens/restuans. Batches were placed into labeled tubes and shipped to the state Arbovirus Laboratory at Wadsworth for viral testing.

\subsection{Viral Testing}

The Arbovirus Laboratory of the Wadsworth Center performed quadraplex quantitative reverse transcriptase-polymerase chain reaction (qRT-PCR) assays against viruses circulating in Central and South America and the Caribbean, including ZIKV, Eastern equine encephalitis virus (EEEV), WNV, along with multiple other flaviviruses present in the state. Detailed descriptions of the plan and operations of the quadraplex qRT-PCR have been published previously [9]. 


\subsection{Statistical Analyses}

Data management and statistical analyses were performed using SAS® statistical software, version 9.4 (SAS Institute Inc., Cary, NC, USA) and Microsoft Excel 2011. During the descriptive analysis, the mosquito count per batch was used as a statistical weight; this was necessary for proper estimation of variance. Differences among mosquito batches between 2016 and pre-existing 2015 surveillance data from comparable trap sites and trap types were estimated via comparison of medians using Bonferroni-adjusted, weighted, non-parametric, rank-transformed, repeatedmeasures ANOVA. These procedures allow analysis for repeated-measures involving samples from heavy-tailed non-normal distributions [10-11]. Statistical significance was set at $\mathrm{p}<0.05$.

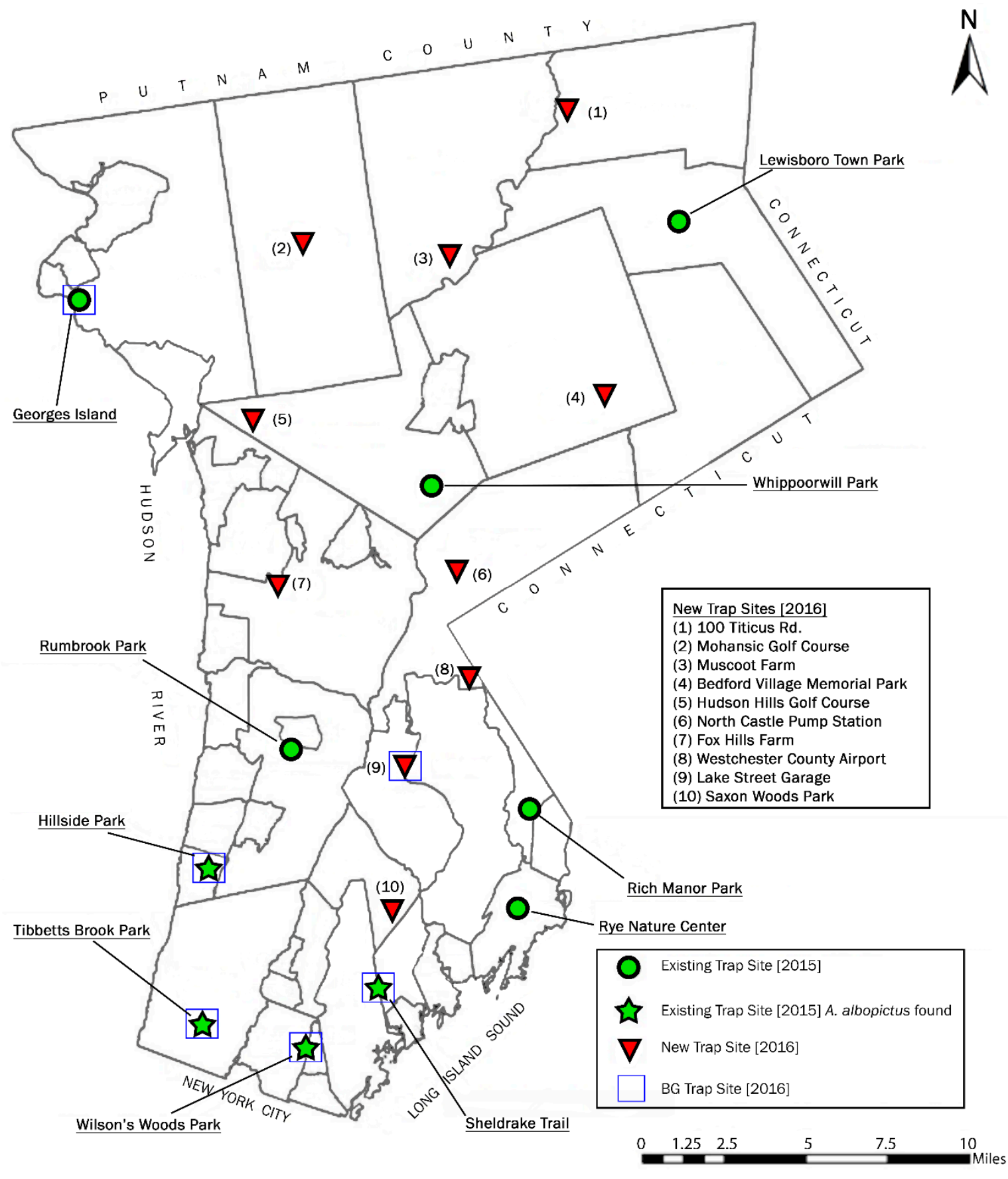

Figure 1. Map of mosquito trap locations in Westchester County, New York, 2016. 


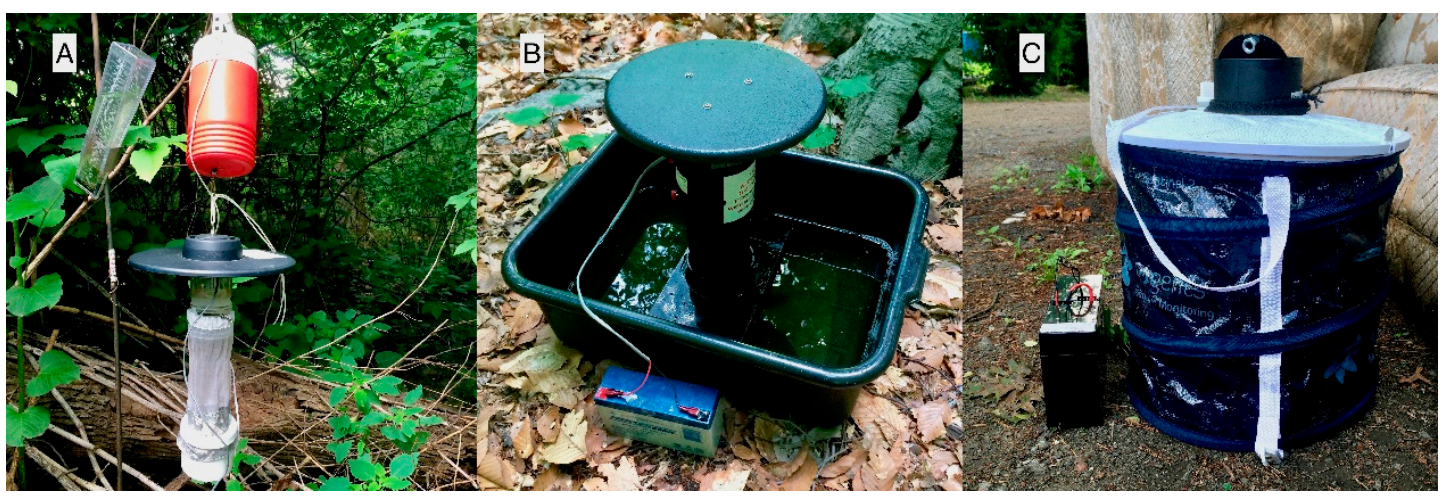

Figure 2. Mosquito traps in Westchester County, New York, 2016. (A) CDC Light Trap, (B) Gravid Trap, (C) BG Trap.

\section{RESULTS}

\subsection{Molecular Screening}

In total, the WCDH sent 369 batches representing 8,891 mosquitoes for laboratory testing, all of which tested negative for ZIKV (Table 1). Seventeen different mosquito species were identified, although only one, A. albopictus, is a known ZIKV vector. A. albopictus represented $80(21.7 \%)$ of the 369 batches sent for viral testing (Table 1). Three positive WNV batches were isolated from Tibbets Brook Park, Yonkers, during August 2016. The first positive WNV batch was isolated from 60 female Cx. salinarius mosquitoes collected by a CDC light trap on August 4, 2016 (Table 1, Figure 1). Six days later, a second positive WNV batch was isolated from 60 female $C x$. pipiens/restuans mosquitoes collected by a Gravid trap on August 11, 2016 (Table 1, Figure 1). Nineteen days later, on August 30, 2016, the final positive WNV batch was isolated from 25 female $C x$. pipiens/restuans mosquitoes collected by a Gravid trap (Table 1, Figure 1).

Table 1. Mosquito collections screened for ZIKV in Westchester County, New York, 2016.

\begin{tabular}{|c|c|c|c|c|c|c|c|c|c|c|}
\hline Species & Batches & Mos. & Id. (No.) & ZIKV & EEEV & WNV & FLAV & Site & $\begin{array}{l}\text { Mosquito } \\
\text { condition }\end{array}$ & $\begin{array}{l}\text { Trap } \\
\text { type }\end{array}$ \\
\hline Culex salinarius & 45 & 1,846 & $\begin{array}{c}16590198 \\
(60)\end{array}$ & - & - & + & - & $\begin{array}{l}\text { Tibbetts } \\
\text { Brook } \\
\text { Park, } \\
\text { Yonkers }\end{array}$ & Normal & $\begin{array}{l}\text { CDC } \\
\text { Light }\end{array}$ \\
\hline \multirow[t]{2}{*}{$\begin{array}{l}\text { Culex pipiens } \\
\text { Irestuans }\end{array}$} & 193 & 5,184 & $\begin{array}{c}16590182 \\
(60)\end{array}$ & - & - & + & - & $\begin{array}{c}\text { Tibbetts } \\
\text { Brook } \\
\text { Park, } \\
\text { Yonkers }\end{array}$ & Gravid & Gravid \\
\hline & & & $\begin{array}{c}16590213 \\
(25)\end{array}$ & - & - & + & - & $\begin{array}{c}\text { Tibbetts } \\
\text { Brook } \\
\text { Park, } \\
\text { Yonkers }\end{array}$ & Gravid & Gravid \\
\hline
\end{tabular}

$\begin{array}{lcc}\text { Aedes albopictus } & 80 & 900 \\ \begin{array}{l}\text { Ochlerotatus } \\ \text { cantator }\end{array} & 2 & 64 \\ \begin{array}{l}\text { Aedes cinereus } \\ \text { Ochlerotatus }\end{array} & 2 & 38 \\ \text { triseriatus } & 2 & 34 \\ \text { Aedes thibaulti } & 1 & 10\end{array}$




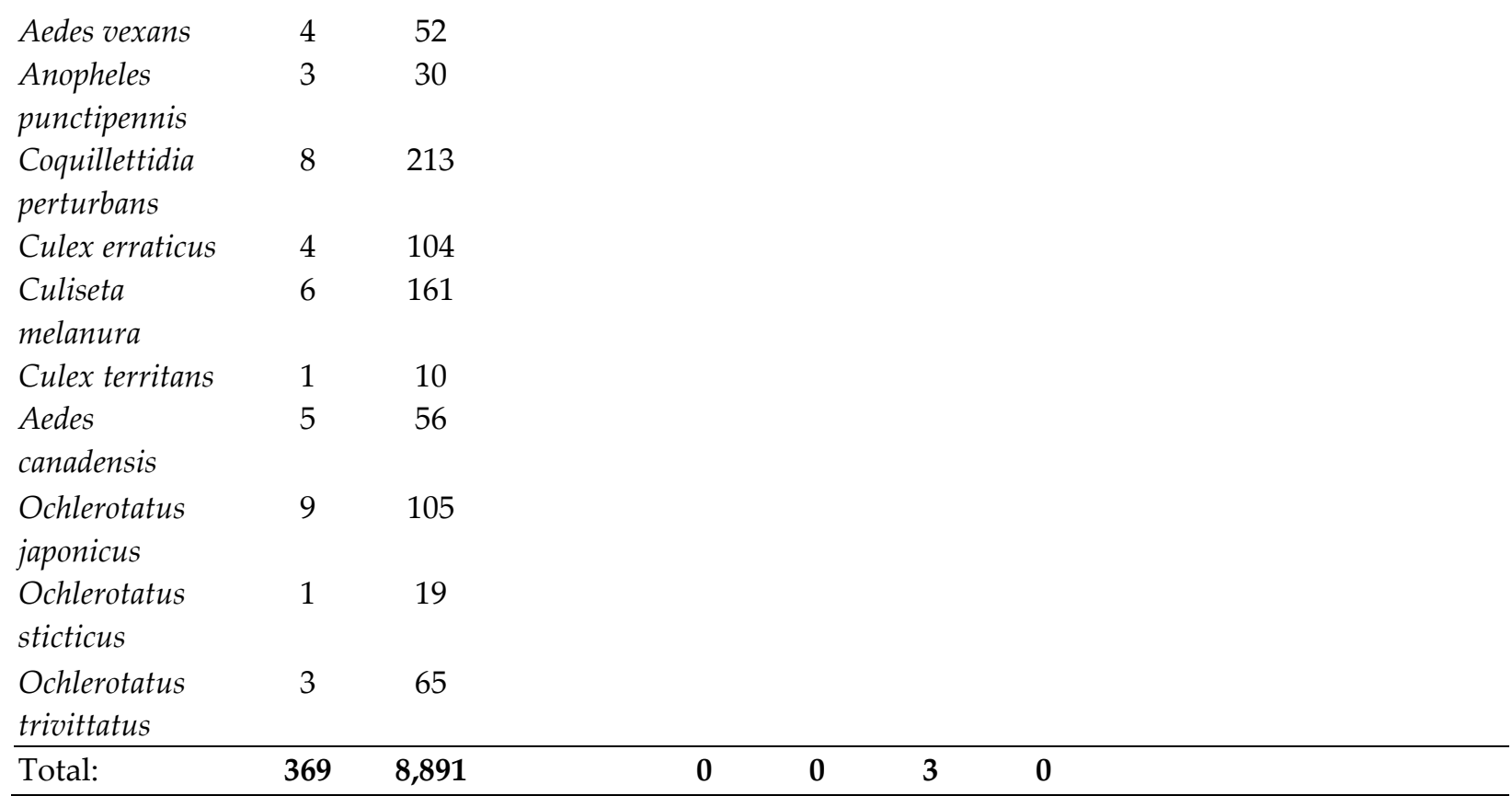

Batches: the total number of individual batches sent for viral testing; Mos.: the total number of individual mosquitoes included in all batches; Id.(No.): mosquito batch positive for virus followed by a total number of mosquitoes included in the batch (given in brackets); FLAV: other flaviviruses.

\subsection{Vector Involvement}

Statistically, the WCDH collected and sent more mosquitoes per batch for testing in 2016 than during 2015 for comparable traps and locations $(p=0.0298)$ (Table 2). The median mosquito count per batch sent for viral testing increased in 2016 (Table 2). Variance increased in 2016, even though the WCDH sent more batches for testing (data not shown). Previously, the WCDH collected viral surveillance information only on WNV and other flaviviruses. The proportion of WNV-positive batches significantly decreased $(\mathrm{p}<0.0001)$ during the 2016 surveillance despite an increase in the number of viral batches sent for testing (Table 2).

Table 2. Crude statistical differences in positive viral batch, collection month, trap type, and mosquito condition between trapping years 2015 and 2016.

\begin{tabular}{lccc}
\hline & $\mathbf{2 0 1 5}$ & $\mathbf{2 0 1 6}$ & p-value \\
\hline Mosquito count per batch, median (IQR) & $35.3(17.9-57.4)$ & $46.5(19.0-59.4)$ & 0.0298 \\
Viral positive Batch, \% (no.) & & & \\
$\quad$ ZIKV & NA & $0.00 \%(0)$ & - \\
$\quad$ EEEV & NA & $0.00 \%(0)$ & - \\
$\quad$ WNV & $7.11 \%(17)$ & $0.81 \%(3)$ & $<0.0001$ \\
$\quad$ FLAV & $1.26 \%(3)$ & $0.00 \%(0)$ & 0.0310 \\
Mosquito count per batch by collection month, & & & \\
median (IQR) & & & \\
$\quad$ June & $43.2(25.0-53.5)$ & $59.0(34.4-59.5)$ & $<0.0001$ \\
$\quad$ July & $27.7(14.2-46.0)$ & $29.0(13.0-58.6)$ & 0.0338 \\
$\quad$ August & $49.2(15.6-55.7)$ & $46.6(16.6-55.3)$ & $\mathrm{NS}$ \\
$\quad$ September & $26.1(14.3-52.3)$ & $28.9(14.4-58.7)$ & $\mathrm{NS}$ \\
Mosquito count per batch by trap type, median & & & \\
(IQR) & & & - \\
$\quad$ BG Trap & $\mathrm{NA}$ & $15.4(10.6-26.3)$ & $<0.0001$ \\
$\quad$ Gravid Trap & $33.4(18.5-51.9)$ & $49.2(22.1-59.4)$ & 0.0009
\end{tabular}




\section{Mosquito count per batch by condition, median (IQR)}

Normal condition

$42.0(17.0-55.5) \quad 37.6(17.3-58.7)$

NS

Gravid condition

$33.2(18.7-51.9)$

$51.7(21.5-59.5)$

$<0.0001$

Blood-fed condition

NA

$13.5(11.0-21.8)$

NS: not statistically significant; NA: no data or insufficient data to complete the analysis.

SD: standard deviation; IQR: interquartile range (Q1-Q3).

FLAV: other flaviviruses.

The WCDH collected and sent more mosquitoes per batch for testing in June and July 2016 than in June and July 2015 ( $p<0.0001$ and $p=0.0338$, respectively; Table 2). Sub-group analysis revealed that this increase might have been attributable to increases in the number of Coquillettidia perturbans and $C x$. pipiens/restuans mosquitoes at Georges Island, Hillside Park, Rich Manor Park, Rye Nature Center, Tibbetts Brook Park, and at Wilson's Woods Park (data not shown). Furthermore, subgroup analysis of mosquito counts per batch revealed that WCDH identified more gravid $C x$. pipiens/restuans specimens in gravid traps at Rumbrook Park and Sheldrake Trail during July 2016 than July 2015 (data not shown). Collectively, the WCDH sent more mosquitoes per batch for testing in both gravid traps $(\mathrm{p}<0.0001)$ and CDC light traps $(\mathrm{p}=0.0009)$ during 2016 surveillance than during the 2015 surveillance (Table 2). Moreover, among all traps, the WCDH collected a significantly greater number of gravid mosquitoes ( $\mathrm{p}<0.0001$ ) in 2016 than in 2015 (Table 2).

Significant increases in mosquito count per batch in 2016 were observed for traps at Tibbetts Brook Park, Rye Nature Center, and Rich Manor Park, which may be attributable to gravid $C x$. pipiens/restuans specimens from gravid traps during June 2016 (data not shown). However, the WCDH collected and sent fewer mosquitoes per batch from Wilson's Woods Park and Sheldrake Trail in 2016 than in 2015. Upon further inspection, sub-group analysis at Wilson's Woods Park and Sheldrake Trail revealed significantly fewer gravid Cx. pipiens/restuans mosquitoes from gravid traps in 2016 than 2015 (data not shown). An internal investigation determined that the batteries that powered these traps at Wilson's Woods Park during July and August 2016 and at Sheldrake Trail in June 2016 experienced power failures. Adjusted, weighted, rank-transformed, non-parametric repeated-measures ANOVA was performed, controlling for traps in 2016 with battery failure and their corresponding traps in 2015. After adjustment, the WCDH still collected and sent more mosquitoes per batch $(\mathrm{p}<0.0001)$ for testing during 2016 than during 2015 (Table 3), however statistical significance increased compared with the crude estimates (Table 2). Likewise, adjusted estimates comparing July 2016 to 2015 mosquito count per batch increased in statistical significance (Table 3). Moreover, the WCDH identified significantly more flaviviruses in 2015 (Table 2). However, after adjustment these differences were eliminated, suggesting that battery failure played a critical role in the failure to collect other flaviviruses at Wilson's Woods Park in 2016 (Table 3). 
Table 3. Adjusted statistical differences in positive viral batch, collection month, trap type, and mosquito condition between trapping years 2015 and 2016.

\begin{tabular}{lccc}
\hline & $\mathbf{2 0 1 5}$ & $\mathbf{2 0 1 6}$ & p-value \\
\hline $\begin{array}{l}\text { Mosquito count per batch, median (IQR) } \\
\text { Viral positive batch, \% (no.) }\end{array}$ & $32.1(15.5-55.8)$ & $47.5(19.0-59.4)$ & $<0.0001$ \\
$\quad$ ZIKV & NA & $0.00 \%(0)$ & - \\
$\quad$ EEEV & NA & $0.00 \%(0)$ & - \\
WNV & $5.61 \%(12)$ & $0.86 \%(3)$ & $<0.0001$ \\
$\quad$ FLAV & $0.00 \%(0)$ & $0.00 \%(0)$ & NS \\
Mosquito count per batch by collection month, & & & \\
median (IQR) & & & \\
$\quad$ June & $37.8(19.5-50.4)$ & $59.1(33.0-59.6)$ & $<0.0001$ \\
$\quad$ July & $24.9(13.5-35.1)$ & $30.5(13.3-59.0)$ & $<0.0001$ \\
$\quad$ August & $47.3(15.0-54.9)$ & $48.5(16.7-55.4)$ & NS \\
$\quad$ September & $26.1(14.3-52.3)$ & $38.9(14.4-58.7)$ & NS \\
Mosquito count per batch by trap type, median & & & \\
(IQR) & & & - \\
$\quad$ BG Trap & $\mathrm{NA}$ & $15.4(10.6-26.3)$ & - \\
$\quad$ Gravid Trap & $28.5(14.7-48.5)$ & $51.8(23.6-59.5)$ & $<0.0001$ \\
$\quad$ CDC Light Trap & $41.7(17.2-55.5)$ & $56.8(18.9-59.0)$ & 0.0009 \\
Mosquito count per batch by condition, & & & \\
median (IQR) & & & NS \\
$\quad$ Normal condition & & & - \\
$\quad$ Gravid condition & & & \\
Blood-fed condition & $42.0(17.0-55.5)$ & $37.6(17.3-58.7)$ & \\
\hline
\end{tabular}

Mosquito batches in 2015 and 2016, adjusted for 2016 battery failure traps. NS: not statistically significant; NA: no data or insufficient data to complete the analysis.

SD: standard deviation; IQR: interquartile range (Q1-Q3); FLAV: other flaviviruses.

Statistical analysis was not performed on the Aedes genus due to a lack of detailed species identification during 2015 surveillance. BG traps began capturing A. albopictus specimens in southern Westchester County from late June through August in 2016 (Figure 3). At the southernmost site, Tibbetts Brook Park, A. albopictus specimens were captured from early June, reaching a peak in late August and mid-September (Figure 3). At Wilson's Woods Park, $3.2 \mathrm{~km}$ to the east, A. albopictus collection steadily increased from mid-July, reaching an apex in early September (Figure 3). One of the new trap sites, located approximately $16 \mathrm{~km}$ north of Tibbets Brook Park at Lake Street Garage, began collecting A. albopictus during mid-August, reaching peak levels of accumulation in late August (Figure 3). This peak was followed by a sudden population decrease in early September, which was in turn followed by another peak in mid-to-late September. No A. albopictus specimens were captured at Hillside Park, even when BG traps were used. This may have been the result of battery inefficiency or failure. Two other trap sites (Rich Manor Park and Rye Nature Center) were able to collect $A$. albopictus with Gravid traps, without BG traps, during August and late September (Figure 3). 


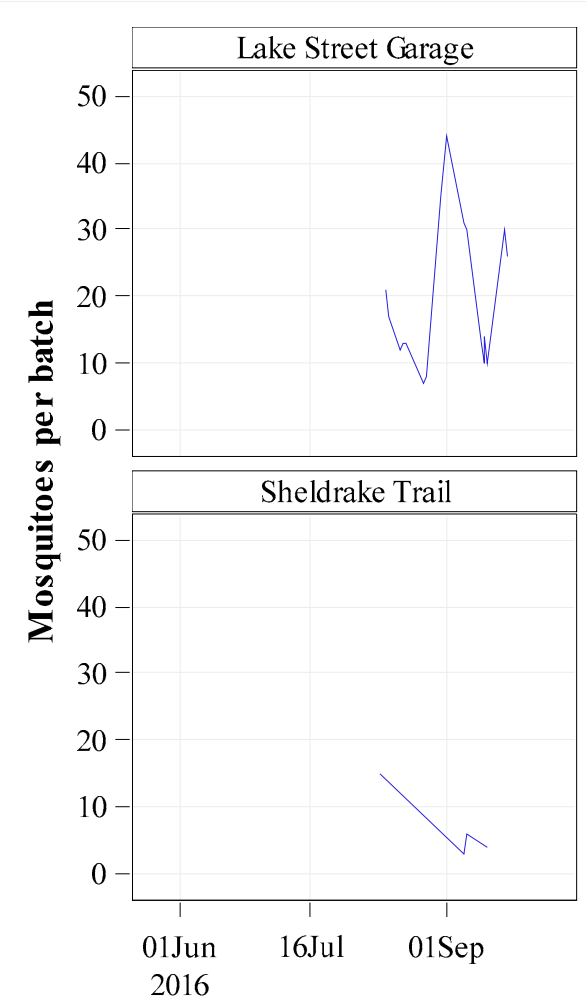

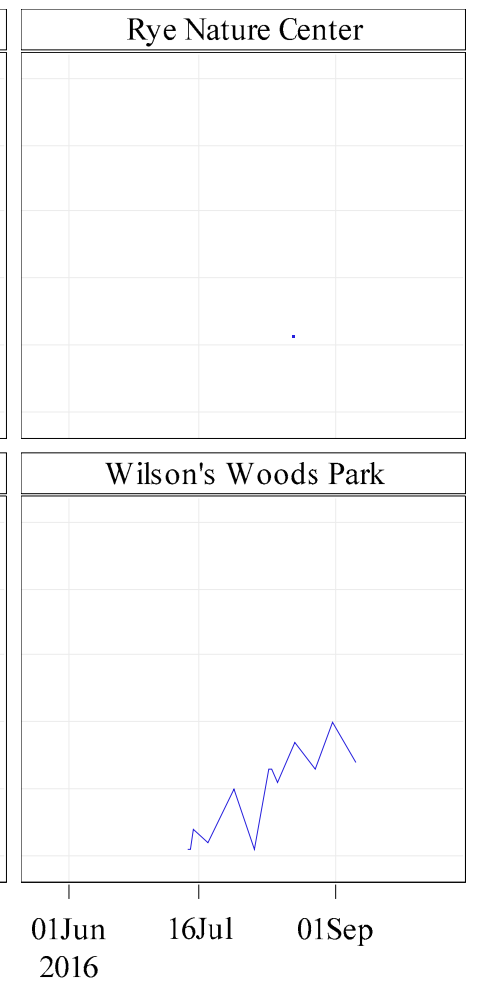

2016

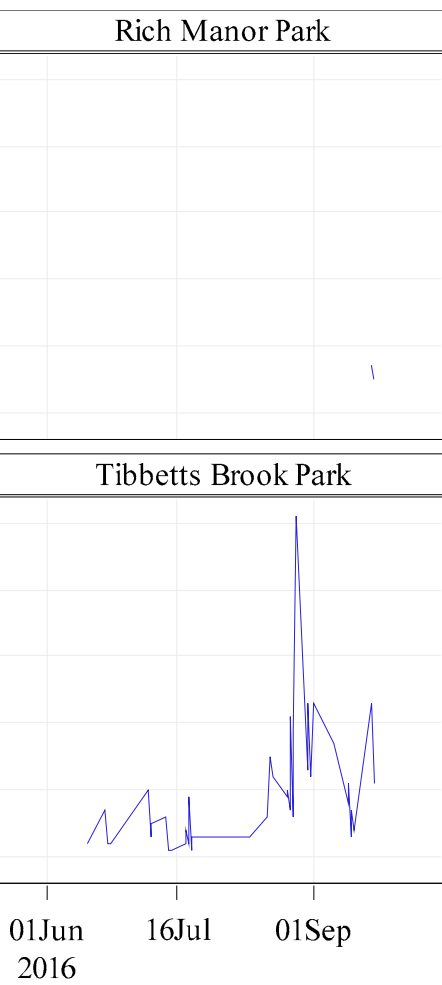

Mos quito collection date

Mosquito species $\longrightarrow$ A. albopictus

Figure 3. A. albopictus batch count according to date and collection site, Westchester County, New York 2016. (top-left) Lake Street Garage; (top-middle) Rich Manor Park; (top-right) Rye Nature Center; (bottomleft) Sheldrake Trail; (bottom-middle) Tibbetts Brook Park; (bottom-right) Wilson's Woods Park.

\section{DISCUSSION}

In November 2016, the State of New York reported 859 cases of Zika; 56 occurred in pregnant women with conclusive laboratory evidence confirming ZIKV infection [12]. All cases reported to the NYSDOH were defined as travel-associated, meaning that such cases were associated with "recent travel to a country or region with known active ZIKV or sexual contact with a recent traveler from these areas." However, this definition does not exclude the possibility that these individuals could have returned home without infection and could have subsequently acquired Zika through local vector-to-human transmission [13]. Most importantly, the NYSDOH Zika testing procedures only included persons who had traveled to an area with Zika virus transmission; it excluded testing for possible cases that were locally acquired [13].

ZIKV was not detected in the mosquitoes captured in Westchester County, despite increased testing capacity. Therefore, it is not possible to determine with certainty the potential for local vectorto-human transmission in the region; even though recent reports showed that $73 \%$ of orally infected A. albopictus were found to have ZIKV in their saliva [14]. Vector potential in the northeast region, specifically basic reproductive numbers, haven't properly been estimated due to the ongoing accumulation of data required to estimate adult female mosquito density correctly. Unsurprisingly, similar findings have been reported in other nations where only human cases of ZIKV infection have been detected [5]. Due to procedural issues related to the strength and lifespan of the batteries used in BG and Gravid traps, the mosquito surveillance results presented should be interpreted conservatively. It is possible that mosquitoes were either infected with or carrying ZIKV, but that the $\mathrm{WCDH}$ was unable to capture such specimens or that Wadsworth Laboratory was unable to 
positively identify these specimens with the primers used. With over 204 known Zika strains in existence and some additional 200 genomes that have not been fully sequenced [15], it is possible that mutations within the probe-binding region could sufficiently lower the sensitivity of the assay [16].

The WCDH had identified two chikungunya, twelve Dengue, and two malaria cases in 2016 [12]. As mentioned previously, the data suggest that the lack of other flaviviruses identified in the 2016 surveillance season may be attributable to the poor battery performance of traps at Wilson's Woods Park. If true, the crude results from the 2016 surveillance season may present misleading information to all persons working in public health. As mentioned in the Results section, A. albopictus appeared around late June, with peak levels observed in August. Future surveillance efforts should focus on increasing the likelihood of $A$. albopictus capture and subsequent virus detection during these months. Unfortunately, BG traps are quite expensive. However, using CDC light traps that have been altered by using BG lures has been reported to be almost as effective as using standard BG traps [17], thus providing a potentially cost-effective method for enhancing surveillance.

\section{CONCLUSION}

In early 2016, the NYSDOH initiated a ZAP, which identified Westchester County as a potential ZIKV vector reservoir region. Failure to identify ZIKV among captured mosquitoes may be attributable to issues related to trapping, specifically battery performance and longevity. These results (or lack thereof) do not fundamentally indicate whether the hypothesized $A$. species is a local ZIKV vector, or whether ZIKV was present or absent in Westchester County during WCDH's 2016 ZAP surveillance. However, effective protective public health actions will depend on the interpretation of the best available data, with prompt initiation of aggressive interventions when necessary. The science regarding ZIKV is rapidly changing, requiring health providers to have access to all available evidence. This study was developed based on the most up-to-date information available, and the findings of the work may thus be subject to modification based on evidence from forthcoming studies.

ACKNOWLEDGMENTS: I would like to thank Mike Condon for unrestricted use and access to previous trapping data at the Westchester County Department of Health (WCDH). I also wish to express my gratitude to Maureen Kennedy for her academic advisement, support, and suggestions. Editorial support, in the form of proofreading, was provided by Editage and funded by the Popper Scholarship of Hudson Valley. Lastly, I wish to thank Alex Boyer, Osato Okundaye, and all those working at the WCDH who have helped to make this study possible.

\section{COMPETING INTEREST: None declared.}

FUNDING: This work was supported by the Westchester County Department of Health. This manuscript presents independent research, and the views expressed are those of the author, which do not necessarily reflect those of the Department of Health. Editorial and open-access fees were supported by the Popper Scholarship of Hudson Valley.

DATA SHARING STATEMENT: Organic surveillance data are archived and remain accessible at the Westchester County Department of Health.

CONTRIBUTION STATEMENT: The sole author was responsible for the conception and design of the study, the acquisition, statistical analysis, interpretation of data, drafting, final editing, and submission of the final manuscript. Additionally, the sole author agrees to be liable for all aspects of the work in guaranteeing that questions related to the precision or integrity of the work are appropriately investigated and resolved.

\section{REFERENCE}

1. Dick, G.; Kitchen, S.; Haddow, A. Zika virus. (I). Isolations and serological specificity. Trans R Soc Trop Med Hyg 1952, 46, 509-520.

2. Marchette, N.J.; Garcia, R.; Rudnick, A. Isolation of Zika virus from Aedes aegypti mosquitos in Malaysia. Am J Trop Med Hyg 1969, 18, 411-415. 
3. Diallo, D.; Sall, A.; Diagne, C.; Faye, O.; Faye, O.; Ba, Y.; et al. Zika virus emergence in mosquitos in southeastern Senegal, 2011. PLoS One 2014, 9, e109442.

4. Grard, G.; Caron, M.; Mombo, I.; Nkoghe, D.; Mboui Ondo, S.; Jiolle, D.; et al. Zika virus in Gabon (Central Africa)-2007: A new threat from Aedes albopictus? PLoS Negl Trop Dis 2014, 8, e2681.

5. Duffy, N.; Chen, T.; Hancock, W.; Powers, A.; Kool, J.; Lanciotti, R.; et al. Zika virus outbreak on Yap Island, Federated States of Micronesia. New Engl J Med 2009, 360, 2536-2543.

6. Broutet, N.; Krauer, G.; Riesen, M.; Khalakdina, A.; Almiron, M.; Aldighieri, A.; et al. Zika virus as a cause of neurologic disorders. New Engl J Med 2016, 374, 1506-1509.

7. Wang, L.; Valderramos, S.; Wu, A.; Ouyang, S.; Li, C.; Brasil, P.; et al. From mosquitos to humans: genetic evolution of Zika virus. Cell Host Microbe 2016, 19, 561-565.

8. Kröckel, U.; Rose, A.; Eiras, A.; Geier, M. New tools for surveillance of adult yellow fever mosquitos: comparison of trap catches with human landing rates in an urban environment. J Am Mosq Control Assoc 2006, 22, 229-238.

9. Zink, S.; Jones, S.; Maffei, J.; Kramer, L. Quadraplex qRT-PCR assay for the simultaneous detection of Eastern equine encephalitis virus and West Nile virus. Diagn Microbiol Infect Dis 2013, 77, 129-132.

10. Zimmerman, D.; Zumbo, B. Relative power of the Wilcoxon test, the Friedman test, and repeated-measures ANOVA on ranks. The Journal of Experimental Education 1993, 62, 75-86.

11. Woodward, M. Epidemiology Study Design and Data Analysis, 3rd ed.; Boca Raton, FL: Chapman \& Hall/CRC Press, 2014.

12. NYSDOH Statewide mosquito-borne disease activity report. 2017. Available online: https://www.health.ny.gov/diseases/west_nile_virus/docs/weekly_arboviral_surveillance_report.pdf. (accessed on 17, 02, 2017)

13. Zika Virus Testing What Local Health Departments Need to Know. 2017. Available online: https://www.health.ny.gov/diseases/zika_virus/docs/2016-02-10_testing_for_lhd_webinar.pdf. (accessed on 17, 02, 2017)

14. Wong P, Li M, Chong C, Ng L, Tan C. Aedes (Stegomyia) albopictus (Skuse): A Potential Vector of Zika Virus in Singapore. PLoS Neglected Tropical Diseases. 2013;7,8, 2348.

15. Virus Pathogen Database and Analysis Resource (ViPR) - Flaviviridae - Genome database with visualization and analysis tools. Viprbrcorg. 2017. Available online: https://www.viprbrc.org/brc/home.spg?decorator=flavi_zika. (accessed on 17, 02, 2017)

16. Brault, A.; Fang, Y.; Dannen, M.; Anishchenko, M, Reisen, W. A naturally occurring mutation within the probe-binding region compromises a molecular-based West Nile Virus surveillance assay for mosquito pools (Diptera: Culicidae). J Med Entomol 2012, 49, 939-941.

17. Urquhart, C.; Paulsen, D.; Moncavo, A.; Trout-Fryxell, R. Evaluating surveillance methods for arboviral vectors of La Crosse virus and West Nile virus of southern Appalachia. J Am Mosq Control Assoc 2016, 32, 24-33.

(C) 2017 by the authors. Licensee Preprints, Basel, Switzerland. This article is an open access article distributed under the terms and conditions of the Creative Commons by Attribution (CC-BY) license (http://creativecommons.org/licenses/by/4.0/). 